\title{
USP7 inhibitor P22077 inhibits neuroblastoma growth via inducing p53-mediated apoptosis
}

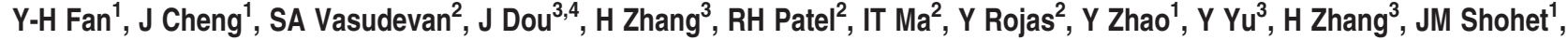 \\ JG Nuchtern², ES Kim ${ }^{2}$ and J Yang,
}

Neuroblastoma (NB) is a common pediatric cancer and contributes to more than $15 \%$ of all pediatric cancer-related deaths. Unlike adult tumors, recurrent somatic mutations in NB, such as tumor protein 53 (p53) mutations, occur with relative paucity. In addition, p53 downstream function is intact in NB cells with wild-type p53, suggesting that reactivation of p53 may be a viable therapeutic strategy for NB treatment. Herein, we report that the ubiquitin-specific protease 7 (USP7) inhibitor, P22077, potently induces apoptosis in NB cells with an intact USP7-HDM2-p53 axis but not in NB cells with mutant p53 or without human homolog of MDM2 (HDM2) expression. In this study, we found that P22077 stabilized p53 by inducing HDM2 protein degradation in NB cells. P22077 also significantly augmented the cytotoxic effects of doxorubicin (Dox) and etoposide (VP-16) in NB cells with an intact USP7-HDM2-p53 axis. Moreover, P22077 was found to be able to sensitize chemoresistant LA-N-6 NB cells to chemotherapy. In an in vivo orthotopic NB mouse model, P22077 significantly inhibited the xenograft growth of three NB cell lines. Database analysis of NB patients shows that high expression of USP7 significantly predicts poor outcomes. Together, our data strongly suggest that targeting USP7 is a novel concept in the treatment of NB. USP7-specific inhibitors like P22077 may serve not only as a stand-alone therapy but also as an effective adjunct to current chemotherapeutic regimens for treating NB with an intact USP7-HDM2-p53 axis.

Cell Death and Disease (2013) 4, e867; doi:10.1038/cddis.2013.400; published online 17 October 2013

Subject Category: Cancer

Neuroblastoma (NB) is an embryonic tumor of the sympathetic nervous system that is derived from neural crest cells. It is the most common extracranial solid tumor in children and accounts for more than $15 \%$ of all pediatric cancer-related deaths. ${ }^{1,2}$ Despite dramatic escalations in therapy over the past years, only modest improvements in overall survival of high-risk NB patients have been achieved. ${ }^{3-6}$ Therefore, novel therapeutic targets and strategies are urgently needed to significantly improve the therapeutic efficacy. Unlike adult tumors, recurrent somatic mutations in NB occur with relative paucity. ${ }^{7}$ It suggests that reactivation of critical tumor suppressor genes, such as tumor protein 53 (p53), may be a viable strategy to treat NB.

The tumor suppressor p53 is one of the most studied and most important regulators of multiple signaling pathways, including normal cellular processes and those triggered by diverse cellular stresses. ${ }^{8-10}$ p53 induces cell growth arrest, apoptosis, and senescence and is mutated in over $50 \%$ of all cancers. ${ }^{11,12}$ In NB, however, p53 mutations are exceedingly rare and occur in less than $2 \%$ of primary tumors. ${ }^{7,13,14}$
Additionally, several studies have shown that p53 downstream functions are intact in NB, and when activated, p53 is capable of inducing normal apoptotic responses. ${ }^{15-17}$ The available evidence highlights that pharmacological reactivation of p53 in NB is a potential and viable strategy to treat NB patients.

In unstressed cells, p53 protein level and activity are maintained at an extremely low level by its primary negative regulator, mouse double minute 2 homolog (MDM2) (human homolog HDM2). ${ }^{18,19}$ MDM2 is an E3 ubiquitin (Ub) ligase that ubiquitinates $\mathrm{p53}$, which results in proteasomal degradation of p53. ${ }^{20,21}$ The effect of MDM2 on p53 has been shown in previous studies in which genetic deletion of MDM2 results in p53 accumulation and subsequent cell death. ${ }^{22-24}$ MDM2, however, is extremely unstable and requires the presence of ubiquitin-specific protease 7 (USP7), an MDM2 de-ubiquitinase, to stabilize it. ${ }^{25}$ USP7 deficiency in mouse embryonic fibroblasts (MEFs) greatly reduces the half-life of MDM2 and activates $p 53 .^{26}$ Taken together, these studies suggest that USP7-MDM2-p53 is a critical axis that functions to limit the

\footnotetext{
${ }^{1}$ Texas Children's Cancer Center, Department of Pediatrics, Dan L Duncan Cancer Center, Baylor College of Medicine, Houston, TX 77030, USA; ${ }^{2}$ Michael E DeBakey Department of Surgery, Division of Pediatric Surgery, Dan L Duncan Cancer Center, Baylor College of Medicine, Houston, TX 77030, USA; ${ }^{3}$ Department of Pathology, University of Texas MD Anderson Cancer Center, Houston, TX 77030, USA and ${ }^{4}$ Xinjiang Key Laboratory of Plant Resources and Natural Products Chemistry, Xinjiang Technical Institute of Physics and Chemistry, Chinese Academy of Sciences, Urumqi, Xinjiang 830011, China

*Corresponding author: J Yang, Texas Children's Cancer Center, Department of Pediatrics, Dan L Duncan Cancer Center, Baylor College of Medicine, Houston, TX 77030, USA. Tel: +832 824 4572; Fax: 832825 4732; E-mail: jianhuay@ bcm.edu

Keywords: neuroblastoma; USP7 inhibitor; P22077; p53; chemotherapy

Abbreviations: NB, neuroblastoma; USP7, ubiquitin-specific protease 7; P22077, 1-(5-((2, 4-difluorophenyl) thio)-4-nitrothiophen-2-yl) ethanone; P53, tumor protein 53; MDM2, mouse double minute 2 homolog; HDM2, human homolog of MDM2; Dox, doxorubicin; VP-16, etoposide; MEFs, mouse embryonic fibroblasts; p21, cyclindependent kinase inhibitor 1; PARP, poly (ADP-ribose) polymerase; Bax, Bcl2-associated X protein; Ub, ubiquitin; PI, propidium iodide; DMSO, dimethyl sulfoxide; SDS, sodium dodecyl sulfate; PAGE, polyacrylamide gel electrophoresis; PVDF, polyvinylidence fluoride; HRP, horse radish peroxidase

Received 01.6.13; revised 23.8.13; accepted 02.9.13; Edited by D Aberdam
} 
level of p53 in cells and that USP7 inhibition can result in p53 activation and cell death.

P22077 has been recently identified, by activity-based chemical proteomics, as an inhibitor of USP7. ${ }^{27}$ P22077 activates p53 and its targeted gene p21 (cyclin-dependent kinase inhibitor 1) in human colon carcinoma HCT116 cells in vitro. ${ }^{27}$ The antitumor effect of P22077 in vivo has not yet been studied. Here, we report that USP7 inhibitor, P22077, potently activates p53 by decreasing HDM2 levels in NB cells with an intact USP7-HDM2-p53 axis and efficiently inhibits tumor growth in vivo. P22077 also enhances chemotherapeutic efficacy and sensitizes chemoresistant NB cells to common chemotherapeutic drugs such as doxorubicin (Dox) and etoposide (VP-16). In addition, high expression of USP7 in NB patients predicts poor outcomes. Collectively, we demonstrate that USP7 is a viable therapeutic target in NB and that the USP7 inhibitor, P22077, can serve as a single agent or as an effective adjunct to current chemotherapeutic regimens in the treatment of NB.

\section{Results}

USP7 inhibitor (P22077) induces apoptosis in a subtype of NB cell lines. To determine the antitumor effect of P22077, we first tested whether P22077 could induce cell death in a panel of NB cell lines including three MYCN-amplified (IMR-32, NGP, and NB-19) and three MYCN non-amplified (CHLA-255, SK-N-AS, and SH-SY5Y) cell lines. P22077 greatly reduced the cell viability of IMR-32, NGP, CHLA-255, and SH-SY5Y cells but not that of NB-19 and SK-N-AS cells (Figure 1a). Microscopic images of cell morphology showed that P22077 dramatically induced cell death in IMR-32 and SH-SY5Y cells but not in SK-N-AS cells (Figure 1b). Furthermore, bioluminescent images of cells also confirmed that P22077 could induce cell death in IMR32 , SH-SY5Y, and NGP cells but not in SK-N-AS cells (Figure 1c). Our results demonstrate that P22077 potently induces cell death in a subset of NB cell lines. We further quantified the percentage of P22077-induced apoptotic cells in the above six NB cell lines. Consistently, we found that P22077 induced apoptosis in IMR-32, SH-SY5Y, NGP, and CHLA-255 cells but not in NB-19 and SK-N-AS cells (Figure 1d and Supplementary Figure S1). Together, P22077 potently induces apoptosis in a subset of NB cell lines, and its effect is independent of MYCN status.

P22077 activates p53 in NB cells with an intact USP7HDM2-P53 axis. To understand the molecular mechanism that determines the sensitivity of NB cells to P22077, we examined the expression level of USP7, HDM2, and p53 in the above six NB cell lines along with LA-N-6 cells, a known chemoresistant NB cell line. All the tested NB cells showed high expression of USP7 (Figure 2a). SK-N-AS cells have a truncated, mutant p53, and NB-19 cells have no detectable HDM2 protein (Figure 2a). ${ }^{28}$ To further determine which cell lines have intact p53 signaling, we treated the cells with Dox, a known p53 inducer and a commonly used chemotherapeutic drug. Dox induced p21 expression in IMR-32, CHLA-255, and SH-SY5Y cells (Figure 2b). Dox also induced $\mathrm{Bcl} 2$-associated $\mathrm{X}$ protein (Bax) expression in all cells except
SK-N-AS cells (Figure 2b). These results suggest that all P22077-sensitive cell lines possess intact p53 signaling and HDM2 expression, whereas in the two P22077-insensitive cell lines, SK-N-AS and NB-19 possesses mutant p53 or lacks expression of HDM2, respectively. Therefore, we conclude that $\mathrm{P} 22077$ sensitivity is determined by the USP7-HDM2-p53 axis in NB cells, and P22077 is capable of inducing apoptosis in NB cell lines with an intact USP7-HDM2-P53 axis.

To further determine the molecular mechanism of P22077induced apoptosis, we examined whether P22077 could stabilize p53 protein by reducing HDM2 stability. As showed in Figures 2c and d, P22077 strongly stabilized p53 protein in p53 wild-type cells (IMR-32 and SH-SY5Y) and was less potent in p53 mutant SK-N-AS cells (Figures 2c and d). Consistently, P22077 strongly suppressed HDM2 protein level in IMR-32 and SH-SY5Y cells but less potent in SK-N-AS cells. P22077 treatment did not affect USP7 protein level in the cells examined (Figures 2c and d). Quantification data from four independent experiments demonstrated that P22077 treatment could significantly stabilize p53 and destabilize HDM2 in IMR-32, SK-N-AS, and SH-SY5Y cells (Figures 2e and f). Furthermore, P22077 strongly induced poly (ADP-ribose) polymerase (PARP) and Caspase-3 cleavage in p53 wild-type IMR-32 and SH-SY5Y cells but not in p53 mutant SK-N-AS cells (Figure $2 \mathrm{~g}$ ). Our results demonstrate that $\mathrm{P} 22077$ induces apoptosis by reactivation of p53 in NB cells with an intact USP7-HDM2-p53 axis.

P22077 significantly enhances the cytotoxic effect of Dox and VP-16 on NB cells. As p53 is activated by genotoxic stress and has a key role in genotoxic stressinduced cell death, we reasoned that pharmacological inhibition of USP7 activity would potentiate p53 activity and result in increased chemosensitivity. To test our hypothesis, IMR-32, SH-SY5Y, and SK-N-AS cells were treated with Dox in combination with or without P22077. P22077 significantly enhanced the cytotoxic effect of Dox on SH-SY5Y and IMR32 cells but not on SK-N-AS cells (Figures $3 a, c$ and e). P22077 also significantly enhanced the cytotoxic effect of VP-16 on SH-SY5Y and IMR-32 cells but not on SK-N-AS cells (Figures $3 b, d$ and $f$ ). To further confirm that our finding was not limited by cell type, we used three more NB cell lines (NGP, NB-19, and CHLA-255) and similar results were observed in all cell lines tested (Supplementary Figure S2). Our results demonstrate that P22077 enhances the cytotoxic effect of Dox and VP-16, and this effect is dependent on an intact USP7-HDM2-P53 axis but independent of MYCN status in NB cells.

P22077 enhances Dox- and VP-16-induced p53-mediated apoptosis. Because p53 activation is critical for genotoxic stress-induced cell death, we reasoned that the potentiated cytotoxic effect of Dox or VP-16 by P22077 may be a result of stabilization of p53 by P22077. To test this hypothesis, we examined whether P22077 could enhance chemotherapyinduced p53 expression in IMR-32 cells. As shown in Figures 4a and b, P22077 enhanced Dox- and VP-16-induced p53 expression in IMR-32 cells. Consistently, Dox and P22077 together significantly increased the cleavage of Caspase-3 
a

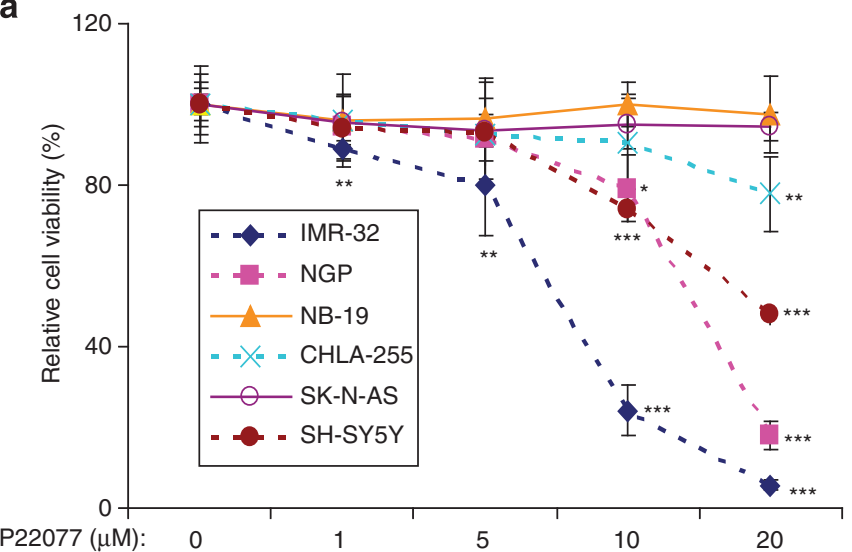

b

IMR-32

SH-SY5Y

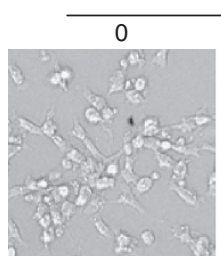

P22077 $(\mu \mathrm{M})$
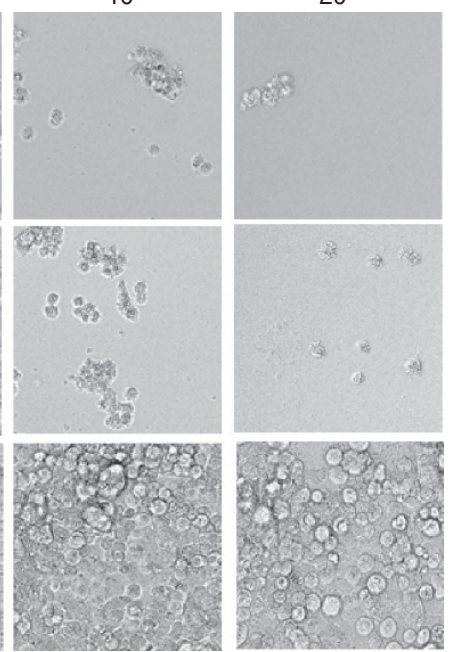

d
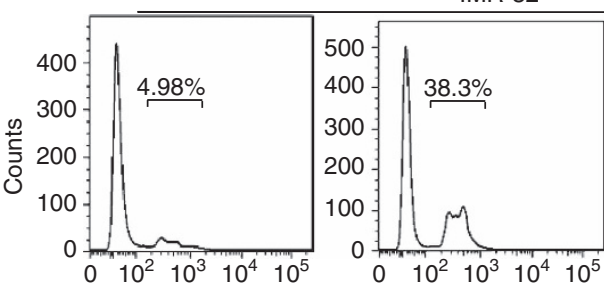

SH-SY5Y
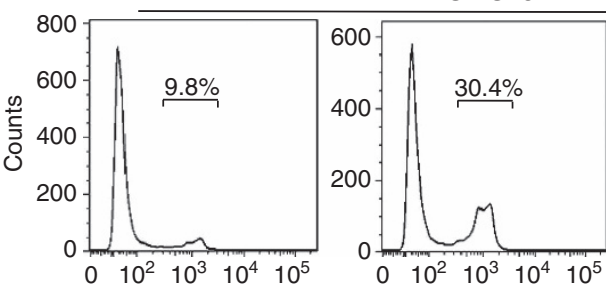

SK-N-AS

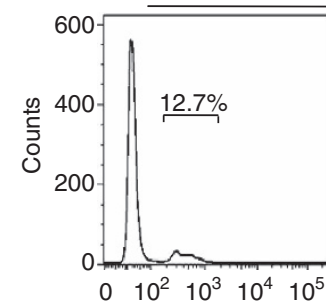

Propidium iodide $(\mathrm{PI})$

P22077 $(\mu \mathrm{M}) \quad 0$

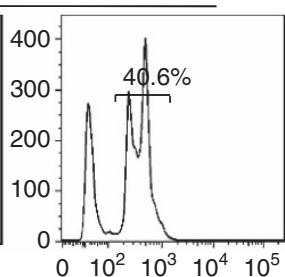

0 1. $10^{2} \quad 10^{3} 10^{4} \quad 10^{5}$
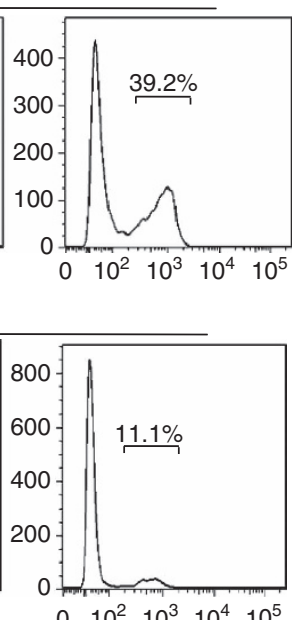

C

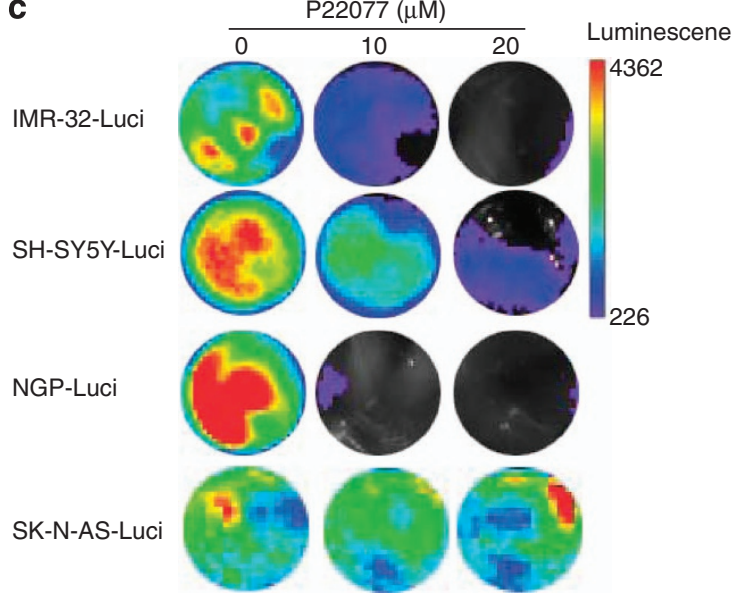

Figure 1 USP7 inhibitor P22077 shows dose-dependent cytotoxic effects in NB cell lines. (a) Cells were seeded in 96-well plates and $24 \mathrm{~h}$ later exposed to P22077 at indicated doses for $24 \mathrm{~h}$. Cell viability was assessed by CCK-8 assay. Results presented as \% vehicle \pm S.D. $(n=6)$. ${ }^{*} P<0.05,{ }^{* *} P<0.01,{ }^{* * *} P<0.001$ (Student's $t$-test). (b) Cells were seeded in six-well plates treated with indicated doses of P22077 for $24 \mathrm{~h}$ and were photographed by using the optical microscope. (c) IMR-32, NGP, SH-SY5Y, and SK-N-AS cell lines with luciferase expression were seeded in 48-well plates, after treatment of P22077 at indicated doses for $24 \mathrm{~h}$, D-luciferin was added to each well for $10 \mathrm{~min}$. Images were taken by bioluminescence imaging instrument. (d) IMR-32, SH-SY5Y, and SK-N-AS cells were treated with 0, 10, and $20 \mu \mathrm{M}$ of P22077 for $24 \mathrm{~h}$ and then apoptotic cells were counted by flow cytometry using PI staining

and PARP in IMR-32 but not in SK-N-AS cells compared with Dox treatment alone (Figures $4 \mathrm{C}$ and $\mathrm{e}$ ). Furthermore, P22077 also increased VP-16-induced Caspase-3 and PARP cleavage in IMR-32 cells (Figure 4d). Our results indicate that P22077 significantly enhances the cytotoxic effect of Dox and VP-16 on NB cells by stabilizing p53.

P22077 sensitizes the chemoresistant LA-N-6 cells to Dox and VP-16-induced apoptosis. Next, we tested whether P22077 could overcome the established chemoresistance in NB cells. The chemoresistant LA-N-6 cell line was treated with equivalent doses of P22077, Dox, and VP16. Consistent with previous reports, LA-N-6 cells were resistant to Dox- and VP-16-induced cell death up to the dose of $10 \mu \mathrm{M}^{29}$ However, $10 \mu \mathrm{M}$ of P22077 caused $\sim 50 \%$ cell death (Figure 5a). Furthermore, P22077 was more potent at inducing apoptosis when compared with Dox and VP-16 (Figure 5b). P22077 could also significantly enhance 
a

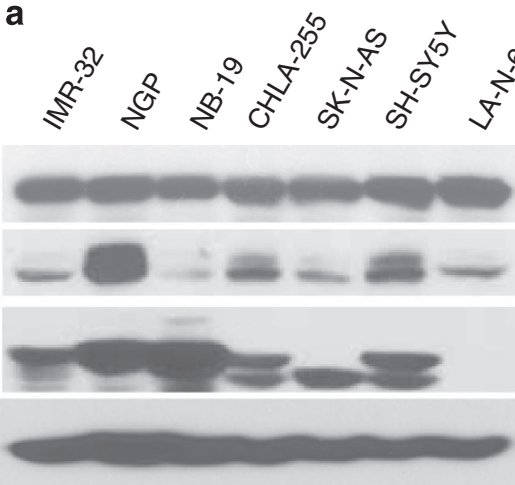

C
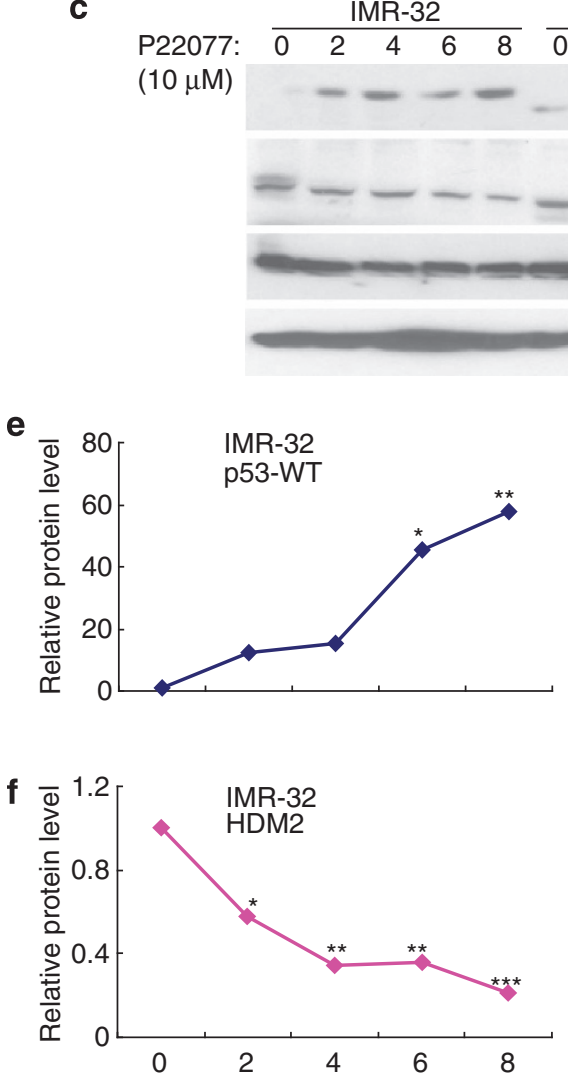

b

\section{con

USP7
HDM2
p53
$\beta$-Actin

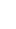

$8 \mathrm{hrs})$

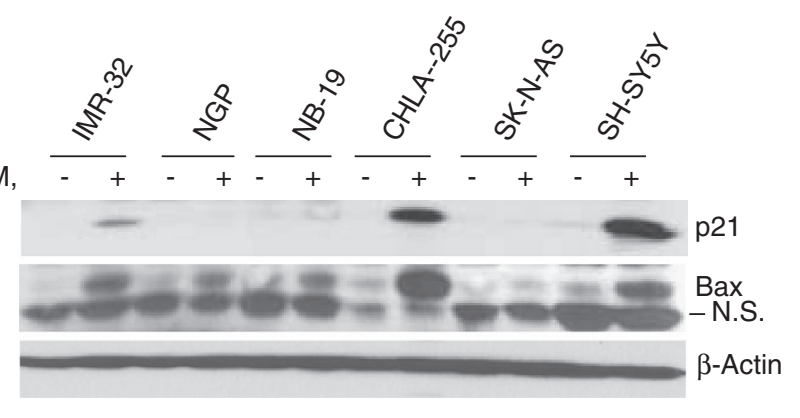

d

(hrs)

p53-WT

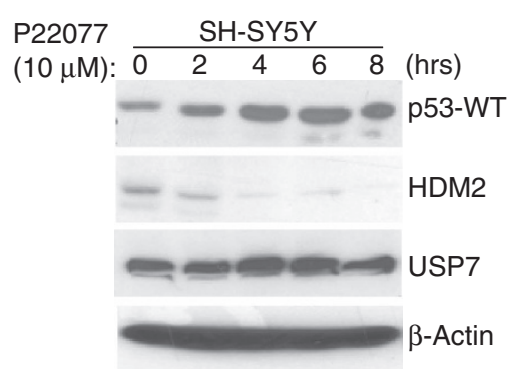

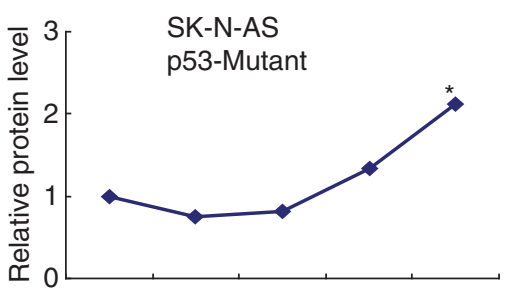
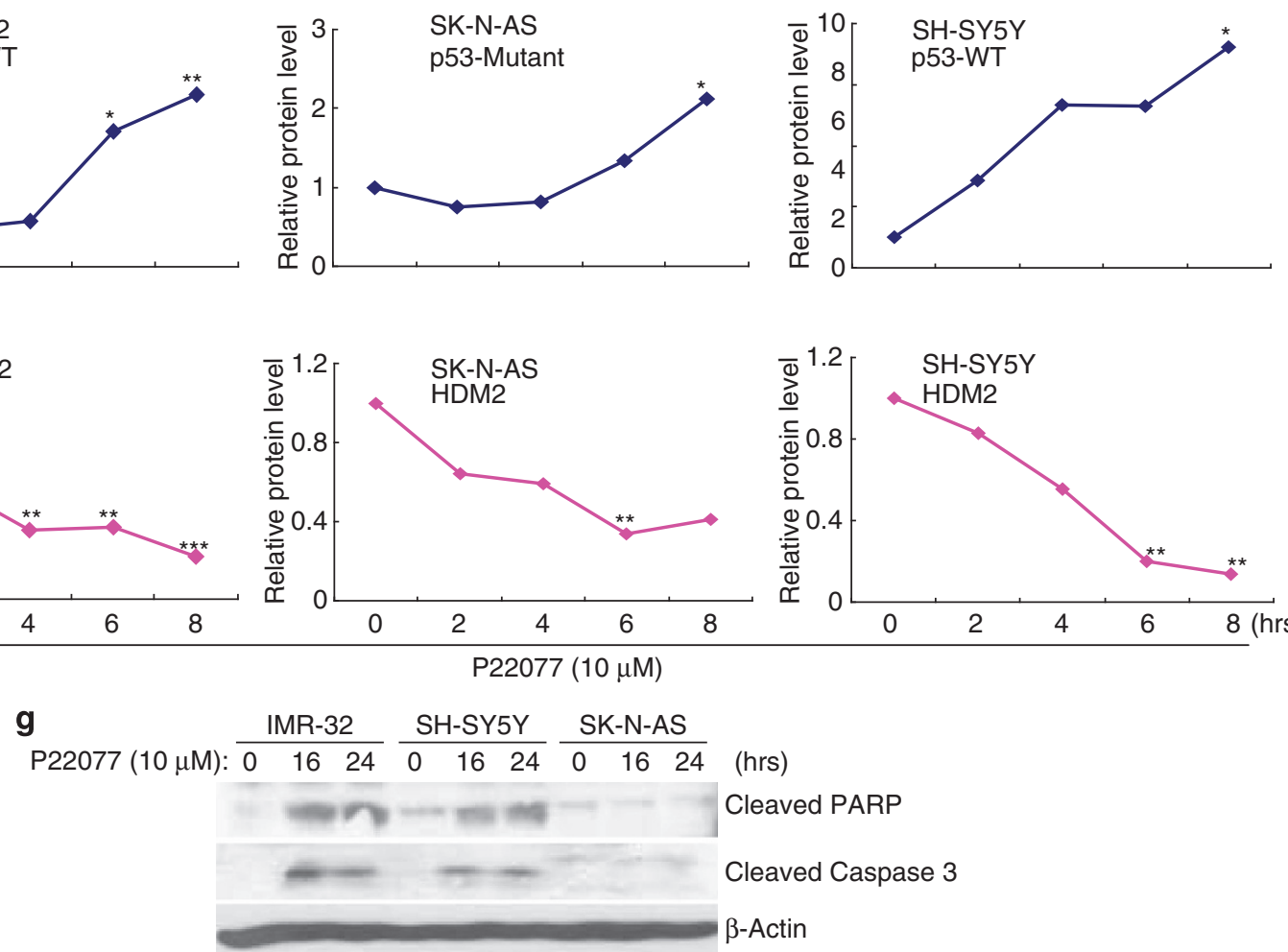

Figure 2 P22077 increases p53 activity and induces apoptosis in p53 wild-type and HDM2-expressing NB cells. (a) The expression of USP7, HDM2, and p53 basal level in seven NB cell lines was detected by WB. (b) NB cells untreated or treated with $1 \mu \mathrm{M}$ of Dox for $8 \mathrm{~h}$ were subjected to SDS-PAGE and immunoblotted with p21 and Bax antibodies. (c and d) IMR-32, SK-N-AS, and SH-SY5Y cells were exposed to $10 \mu \mathrm{M}$ of P22077 for varying durations (0-8h) and then subjected to SDS-PAGE and immunoblotted with p53, HDM2, and USP7 antibodies. (e and f) Quantification of p53 (e) and HDM2 (f) protein level from four independent experiments. (g) IMR-32, SH-SY5Y, and SK-N-AS cells were exposed to $10 \mu \mathrm{M}$ of P22077 for varying durations $(0,16$, and $24 \mathrm{~h})$ and then subjected to SDS-PAGE and immunoblotted with PARP and Caspase-3 antibodies. $\beta$-Actin antibodies were used as a loading control 
a

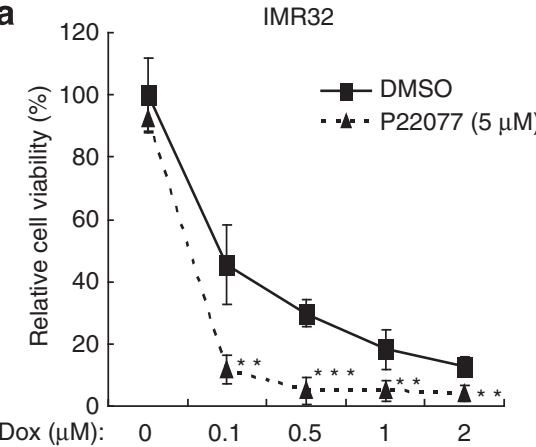

C

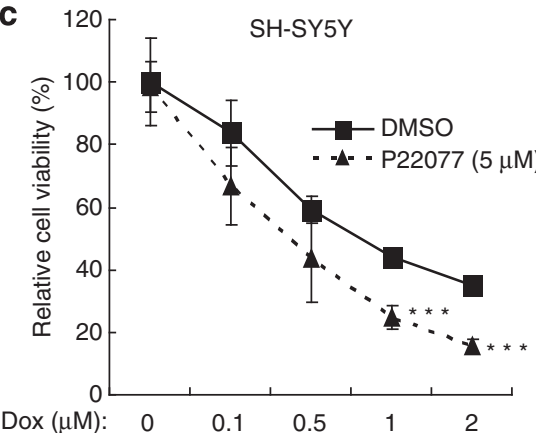

e

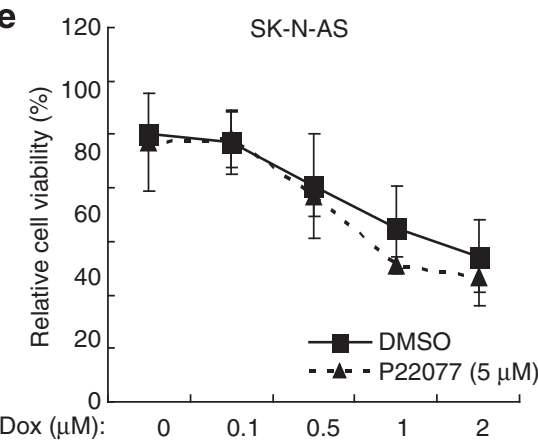

b
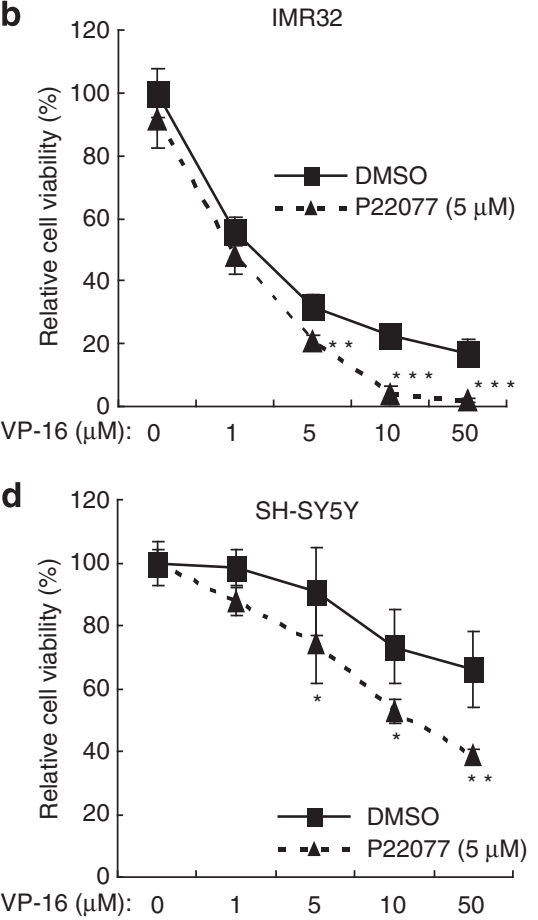

f

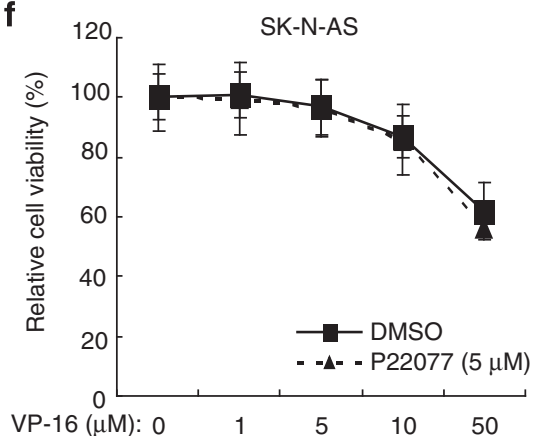

Figure 3 P22077 enhances Dox- and VP-16-induced inhibition of cell proliferation in p53 wild-type NB cell lines. (a, c and e) IMR-32, SH-SY5Y, and SK-N-AS cells were seeded in 96-well plates and after $24 \mathrm{~h}$ of incubation with increasing concentrations of Dox $(0,0.1,0.5,1$, and $2 \mu \mathrm{M})$ plus DMSO or $5 \mu \mathrm{M}$ of P22077, proliferation was measured by a CCK-8 assay. (b, $\mathbf{d}$ and $\mathbf{f}$ ) IMR-32, SH-SY5Y, and SK-N-AS cells were seeded in 96-well plates and after $24 \mathrm{~h}$ of incubation with increasing concentrations of VP-16 $(0,1,5,10$, and $50 \mu \mathrm{M})$ plus DMSO or $5 \mu \mathrm{M}$ of P22077, proliferation was measured by a CCK-8 assay. Results presented as $\%$ vehicle \pm S.D. $(n=6)$. ${ }^{*} P<0.05$, ${ }^{* *} P<0.01,{ }^{* * *} P<0.001$ (Student's $t$-test)

Dox- and VP-16-induced cell death in LA-N-6 cells (Figures 5c and d and Supplementary Figure S3). P22077 increased $\mathrm{p} 53$ protein level and suppressed HDM2 protein level in LA-N-6 cells (Figure 5e). In addition, P22077 induced a much higher p53 protein level compared with Dox and VP-16 in LA-N-6 cells (Figure 5f). However, we did not observe obvious p53 mRNA induction after P22077 treatment when compared with Dox treatment in LA-N-6 cells (Figure $5 \mathrm{~g}$ ). Taken together, these results indicate that P22077 can overcome the established chemoresistance in NB cells by reactivating p53.

P22077 significantly inhibits NB tumor growth in vivo. Next, we tested whether USP7 inhibition by P22077 could inhibit NB tumor growth in vivo. We utilized an orthotopic NB mouse model by surgical injection of IMR-32 cells with luciferase expression into the left renal capsule of nude mice. Two weeks after injection, tumor signals were detected by bioluminescent imaging. Mice bearing tumors were randomly divided into two groups and treated with dimethyl sulfoxide (DMSO) (control) or P22077. P22077 was administered alone at $15 \mathrm{mg} / \mathrm{kg}$ daily for 3 weeks. Treatment with P22077 significantly inhibited tumor growth when compared with control (Figures $6 a$ and b). We observed similar results using other two NB cell lines, SH-SY5Y and NGP, in the orthotopic NB mouse models (Figures 6c-f). Of note, there were no obvious health problems or weight loss of mice in either the control or treatment group during the study (Supplementary Figure S4). Our results demonstrate that P22077 is a potent antitumor drug for treating NB with an intact USP7-HDM2-p53 axis in the mouse model.

High expression of USP7 predicts poor outcome in NB patients. P22077-mediated inhibition of NB growth in vitro 


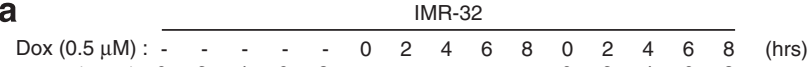
P22077 $(5 \mu \mathrm{M}):: 0 \begin{array}{llllllllllllllll}0 & 2 & 4 & 6 & 8 & - & - & - & - & - & 0 & 2 & 4 & 6 & 8 & \text { (hrs) }\end{array}$

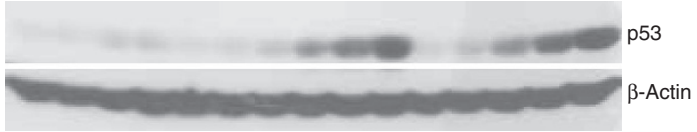

C

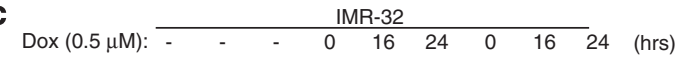

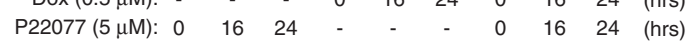

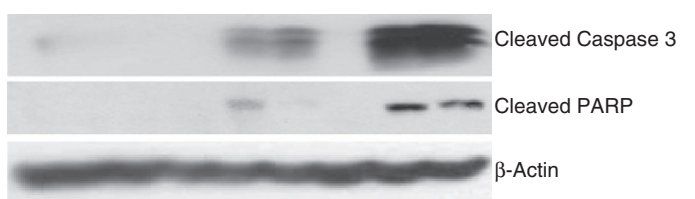

b VP-16 $(10 \mu \mathrm{M}):$\begin{tabular}{llllllllllllllll}
\hline & - & - & - & - & 0 & 2 & 4 & 6 & 8 & 0 & 2 & 4 & 6 & 8 & (hrs)
\end{tabular} $\mathrm{P} 22077(5 \mu \mathrm{M}): 0 \begin{array}{llllllllllllllll}0 & 2 & 4 & 6 & 8 & - & - & - & - & - & 0 & 2 & 4 & 6 & 8 & \text { (hrs) }\end{array}$

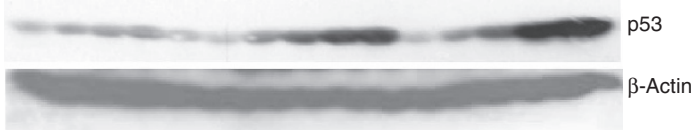

d

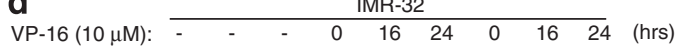

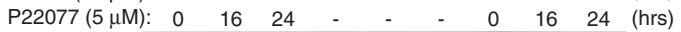

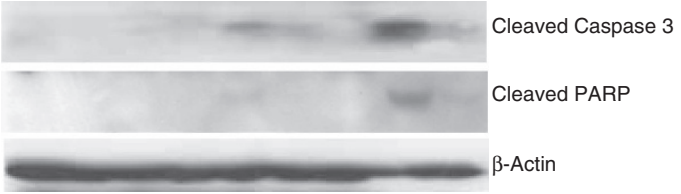

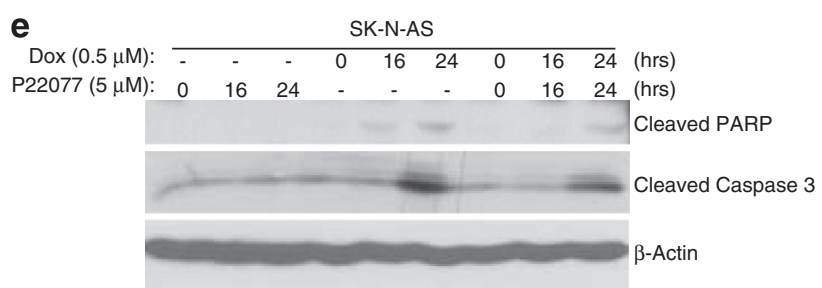

Figure 4 P22077 enhances Dox- and VP-16-induced apoptosis in p53 wild-type NB cell line. (a and c) IMR-32 cells were treated with either Dox (0.5 $\mu$ M) alone, P22077 $(5 \mu \mathrm{M})$ alone, or a combination for varying durations, subjected to SDS-PAGE, and immunoblotted with p53, PARP, and Caspase-3 antibodies. (b and d) IMR-32 cells were treated with either VP-16 (10 $\mu \mathrm{M})$ alone, P22077 (5 $\mu \mathrm{M})$ alone, or combination for varying durations, then subjected to SDS-PAGE, and immunoblotted with p53, PARP, and Caspase-3 antibodies. (e) SK-N-AS cells were treated with either Dox $(0.5 \mu \mathrm{M})$ alone, P22077 (5 $\mu \mathrm{M})$ alone, or combination for varying durations, then subjected to SDSPAGE, and immunoblotted with PARP or Caspase-3 antibodies

and in vivo demonstrates that USP7 is a viable target for the treatment of NB. We examined whether USP7 expression can be used to predict outcomes of NB patients. Data analysis in the R2 database (R2: http://r2.amc.nl) shows that high expression of USP7 significantly predicts poor outcome in the Versteeg-88 data set $(P=0.01)$ (Figure 7a). This is consistent with results of MYCN non-amplified NB patients from the Seeger-102 data set $(P=0.018)$ (Figure $7 \mathrm{~b})$. These findings suggest that USP7 is a potential biomarker for the prediction of outcomes in NB patients.

\section{Discussion}

The activation of genomic guardian p53 can induce tumor cell death, and the identification of small molecules allowing p53 reactivation is one of the most actively explored fields in cancer research. ${ }^{30,31}$ This is particularly remarkable for NB therapy due to the fact that the majority of NB tumors have wild-type p53 and its intact downstream pathway. ${ }^{7,13,14}$ Here, we report that USP7 inhibitor P22077 is a potent p53 reactivator in NB cells that possess an intact USP7-HDM2p53 axis. In addition, P22077 also significantly improves the efficacy of currently used chemotherapeutic drugs and overcomes the established chemoresistance in NB cells in vitro. P22077 inhibits NB tumor growth in an orthotopic mouse model. Collectively, we have demonstrated that inhibition of USP7 by a small-molecule inhibitor P22077 can result in reactivation of p53 and consequently inhibit tumor growth. All of the tested seven NB cell lines show high expression of USP7 protein, indicating that USP7 is an ideal target in NB. This is further supported by the microarray analysis data with NB patient tumor samples, showing that high expression of USP7 predicts poor outcomes. Taken together, we conclude that targeting USP7 by small-molecule inhibitors such as P22077 is a novel therapeutic concept in NB.

Several compounds that target HDM2 have been shown to be able to reactivate $p 53 .{ }^{30,31}$ These include small molecules such as Nutlin-3, which blocks the interaction of p53 with MDM2; HLI98, which targets the Ub ligase activity of MDM2. ${ }^{32-35}$ RG7112 is an analog of Nultin-3a and has been tested in clinical trials for the treatment of advanced solid tumors and hematologic neoplasms (www.clinicaltrials.gov, NCT00559533, NCT00623870). These approaches support efforts to treat human cancers by the way of pharmacological reactivation of $p 53$. Here, we show that inhibition of USP7 by a small-molecule inhibitor P22077 is an alternative and efficient approach to reactivate $\mathrm{p53}$ in NB cells. MDM2 acts as a primary negative regulator of $\mathrm{p} 53$ by two major ways: (1) through ubiquitination of p53 to promote proteasomemediated degradation; and (2) by directly binding to p53 and masking its transactivation domain. ${ }^{18,36}$ Therefore, it is very likely that pharmacological inhibition of USP7, a master de-ubiquitinase that controls MDM2 stability, is more efficient than the inhibition of either MDM2/p53 interaction or MDM2-E3 ligase activity alone.

USP7 has a key role in regulating the level of p53 protein and is one of the most interesting de-ubiquitinating enzymes from a therapeutic perspective. ${ }^{37,38}$ However, pharmacological inhibition of USP7 has been achieved only 
a
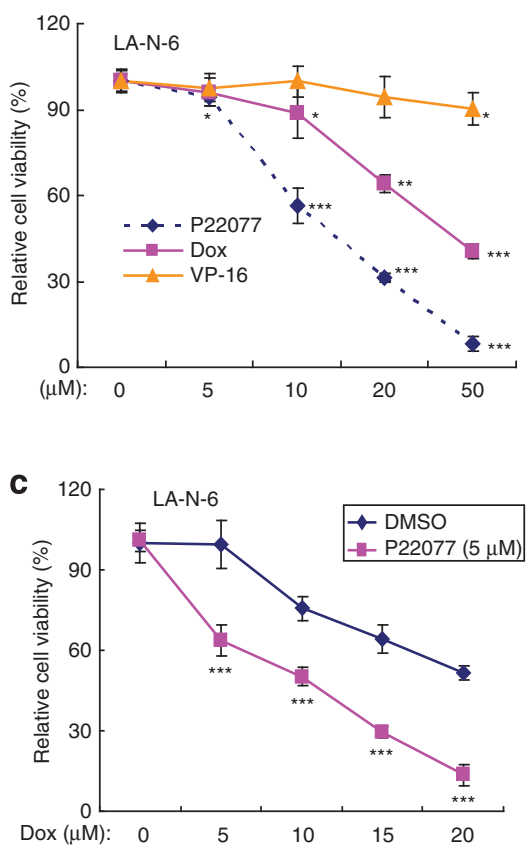

b

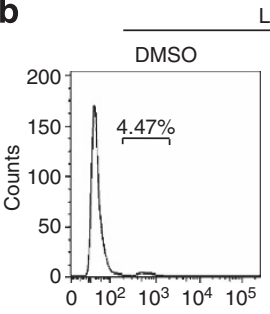

LA-N-6

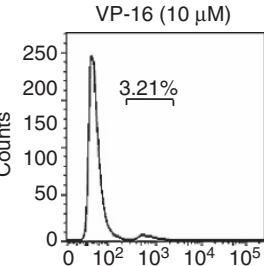

$\operatorname{Dox}(10 \mu \mathrm{M})$

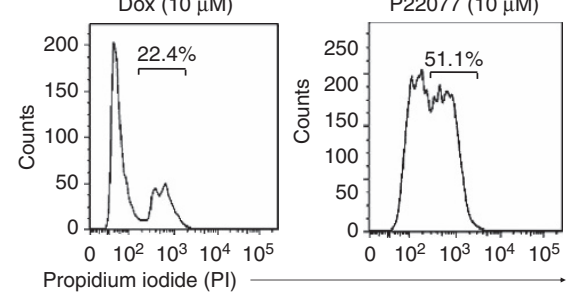

e

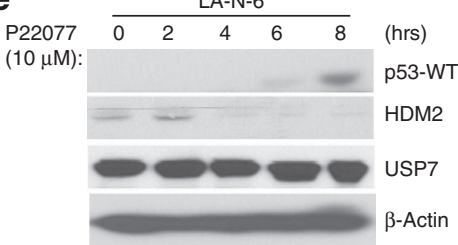

f

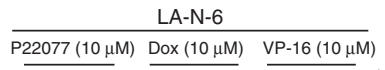

$\frac{\mathrm{P} 22077(10 \mu \mathrm{M})}{068} \frac{\mathrm{Dox}(10 \mu \mathrm{M})}{068} \frac{\mathrm{VP}-16(10 \mu \mathrm{M})}{068}$ (hrs)

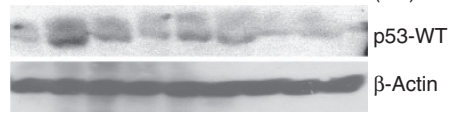

g

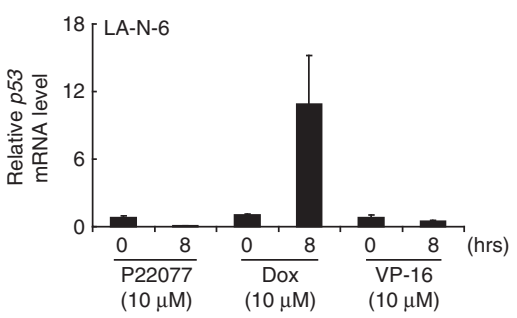

Figure 5 P22077 induces apoptosis in chemoresistant LA-N-6 cell line. (a) LA-N-6 cells were seeded in 96-well plates and after $24 \mathrm{~h}$ of incubation with increasing concentrations of Dox, VP-16, or P22077 (0,5, 10,20, and $50 \mu \mathrm{M})$, cell proliferation was measured by a CCK-8 assay. (b) LA-N-6 cells were treated with $10 \mu \mathrm{M}$ of Dox, VP-16, or P22077 for $24 \mathrm{~h}$ and apoptotic cells were counted by flow cytometry using PI staining. (c) LA-N-6 cells were treated with either Dox alone, P22077 alone, or a combination for varying doses, cell proliferation was measured by a CCK-8 assay. (d) LA-N-6 cells were treated with either VP-16 alone, P22077 alone, or a combination for varying doses, proliferation was measured by a CCK-8 assay. (e) LA-N-6 cells were treated with P22077 $(10 \mu \mathrm{M})$ for a varying duration $(0-8 \mathrm{~h})$ and subjected to SDS-PAGE and immnoblotted with p53, HDM2, and USP7 antibodies. $\beta$-Actin antibodies were used as a loading control. (f) LA-N-6 cells were treated with P22077 (10 $\mu \mathrm{M})$, Dox (10 $\mu \mathrm{M})$, or VP-16 $(10 \mu \mathrm{M})$ with different duration and subjected to SDS-PAGE, and immnoblotted with p53 antibodies. $\beta$-Actin antibodies were used as a loading control. (g) LA-N-6 cells were treated with P22077 $(10 \mu \mathrm{M})$, Dox $(10 \mu \mathrm{M})$ or VP-16 $(10 \mu \mathrm{M})$ with different duration and subjected to Real-time RT-PCR for quantification of P53 mRNA. Results presented as \% vehicle \pm S.D. $(n=6) .{ }^{*} P<0.05,{ }^{* \star} P<0.01,{ }^{* \star *} P<0.001$ (Student's $t$-test)

recently. To our knowledge, there are five small-molecular inhibitors (HBX42108, HBX19818, HBX28258, P5091, and P22077) and one Ub variant (U7Ub25.2540) that have been reported to inhibit USP7 in cells. ${ }^{27,39-42}$ Among these inhibitors, only P5091 has been tested in vivo and has been shown to inhibit multiple myeloma proliferation. ${ }^{39}$ Our data demonstrate that P22077 is a potent USP7 inhibitor and can efficiently induce p53-mediated apoptosis in NB cells with an intact USP7-HDM2-p53 axis and inhibit NB growth in vivo.
One of the possible caveats of USP7 inhibitor for tumor treatment is its potential toxicity. Genetic deletion of USP7 in mice results in early embryonic death between embryonic days E6.5 and E7.5. This suggests that USP7 is required for mouse development in the embryonic stage. In our treatment strategy, mice treated with P22077 $(15 \mathrm{mg} / \mathrm{kg})$ on a daily schedule for 3 weeks or $20 \mathrm{mg} / \mathrm{kg}$ on a daily schedule for 12 days did not show obvious health problems or weight loss in our in vivo model. The in vivo treatment using another USP7 inhibitor, P5091 (20 mg/kg), on a twice-weekly schedule for 
a

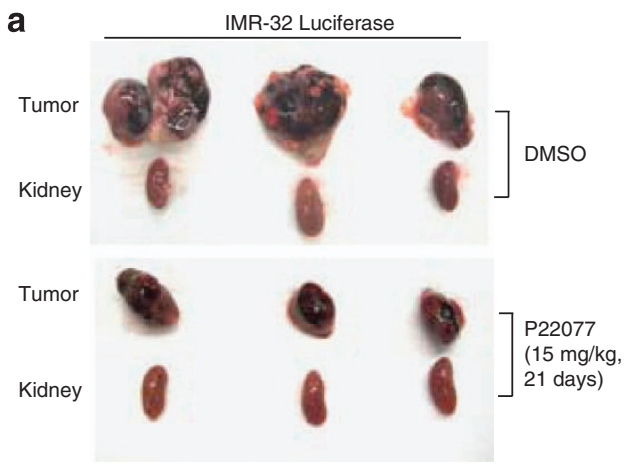

C

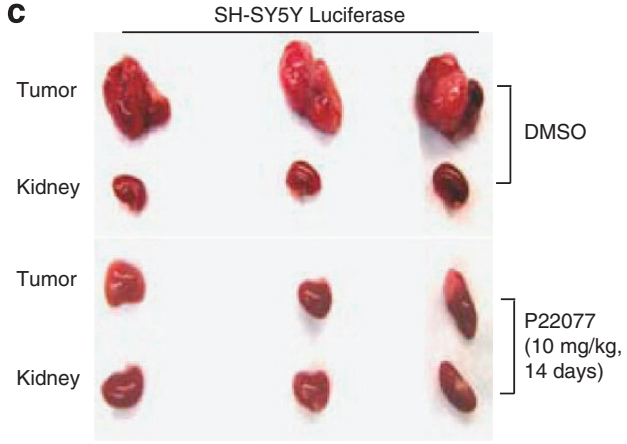

e

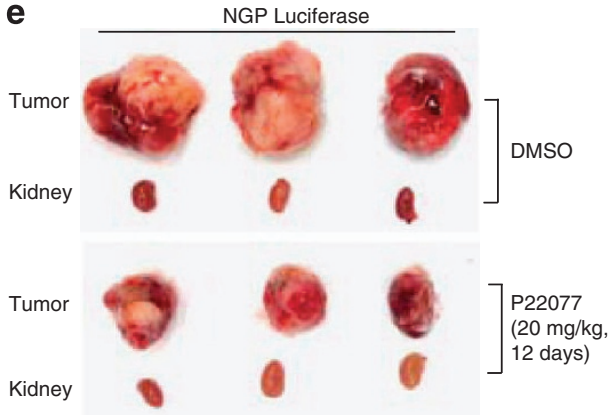

b

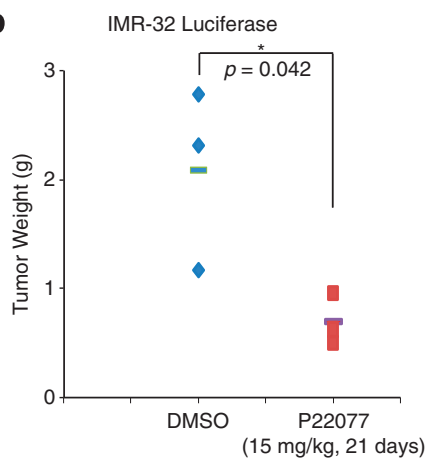

d
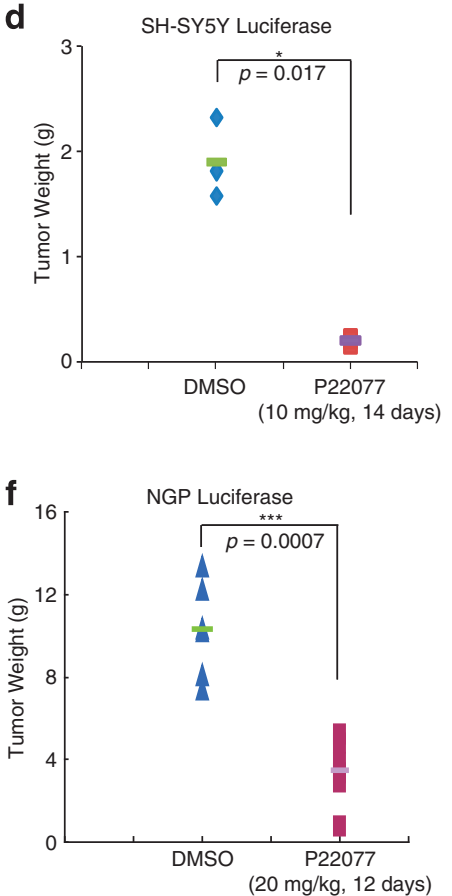

Figure 6 P22077 inhibits NB tumor growth in an orthotopic xenograft mouse model. (a) Photos of IMR-32-derived tumors and corresponding right sided kidney controls from DMSO control group and P22077 treatment group (15 mg/kg) at the end of treatment (21 days). (b) IMR-32-derived tumor weights from the end of treatment presented as the mean with SDs. ${ }^{*} P<0.05$ (Student's $t$-test; one tailed). (c) Photos of SH-SY5Y-derived tumors and corresponding right sided kidney controls from DMSO control group and P22077 treatment group (10 mg/kg) at the end of treatment (14 days). (d) SH-SY5Y-derived tumor weights from the end of treatment presented as the mean with SDs. ${ }^{*} P<0.05$ (Student's $t$-test; two-tailed). (e) Photos of NGP-derived tumors and corresponding right side kidney controls from DMSO control group and P22077 treatment group $(20 \mathrm{mg} / \mathrm{kg})$ at the end of treatment (12 days). (f) NGP-derived tumor weights from the end of treatment presented as the mean with SDs. ${ }^{* \star *} P<0.001$ (Student's $t$-test; two-tailed)

3 weeks did not show weight loss either. ${ }^{39}$ The very limited in vivo data suggest that pharmacological inhibition of USP7 after the embryonic stage may be safe. However, more in vivo data with USP7 inhibitors and analysis of the effect of USP7 genetic deletion on mice after birth are required to determine the safety of targeting USP7 with its small-molecule inhibitors.

In summary, a small molecule, P22077 inhibits the function of USP7 resulting in p53 reactivation in NB cells (Figure $7 \mathrm{c}$ ). Our preclinical studies provide the rationale for the development of de-ubiquitinase-based therapies for NB and specifically demonstrate the promise of therapeutics targeting USP7 to improve the outcome of NB patients. NB patients with an intact USP7-HDM2-p53 axis may benefit from P22077 treatment either as single antitumor drug or as an effective adjunct to current chemotherapeutic regimens (Figure 7c).

\section{Materials and Methods}

Reagents and antibodies. P22077 [1-(5-((2, 4-difluorophenyl) thio)-4nitrothiophen-2-yl) ethanone] was purchased from EMD Millipore (662142) (EMD Millipore, Billerica, MA, USA). Anti-PARP (9532 S), anti-Caspase-3 (9662 S), anti-Mouse (7076 S), and anti-Rabbit (7074 S) antibodies were purchased from Cell Signaling (Cell Signaling Technology, Danvers, MA, USA). Anti-p53 (sc-126), anti-HDM2 (sc-813), anti-p21 (sc-53870), and anti-Bax (sc-493) were purchased from Santa Cruz Biotechnology (Santa Cruz Biotechnology, Dallas, TX, USA). Anti-USP7 (A300-033 A) antibodies were purchased from Bethyl (Bethyl Laboratories, Montgomery, TX, USA). Anti- $\beta$-Actin (A2228) antibodies, Dox (D1515), and etoposide (VP-16, E1383) were purchased from Sigma (Sigma-Aldrich Corp, St. Louis, MO, USA).

Cell lines and cell culture. The human NB cell lines IMR-32, NGP, NB-19, CHLA-255, SK-N-AS, and SH-SY5Y were maintained in RPMI 1640 (Cellgro, Manassas, VA, USA) supplemented with 10\% heat-inactivated fetal bovine serum (FBS) (SAFC Biosciences, Lenexa, KS, USA), 100 units/ml penicillin, $100 \mu \mathrm{g} / \mathrm{ml}$ streptomycin, and $2 \mathrm{mM}$ glutamine. The LA-N-6 cell line was maintained in RPMl 

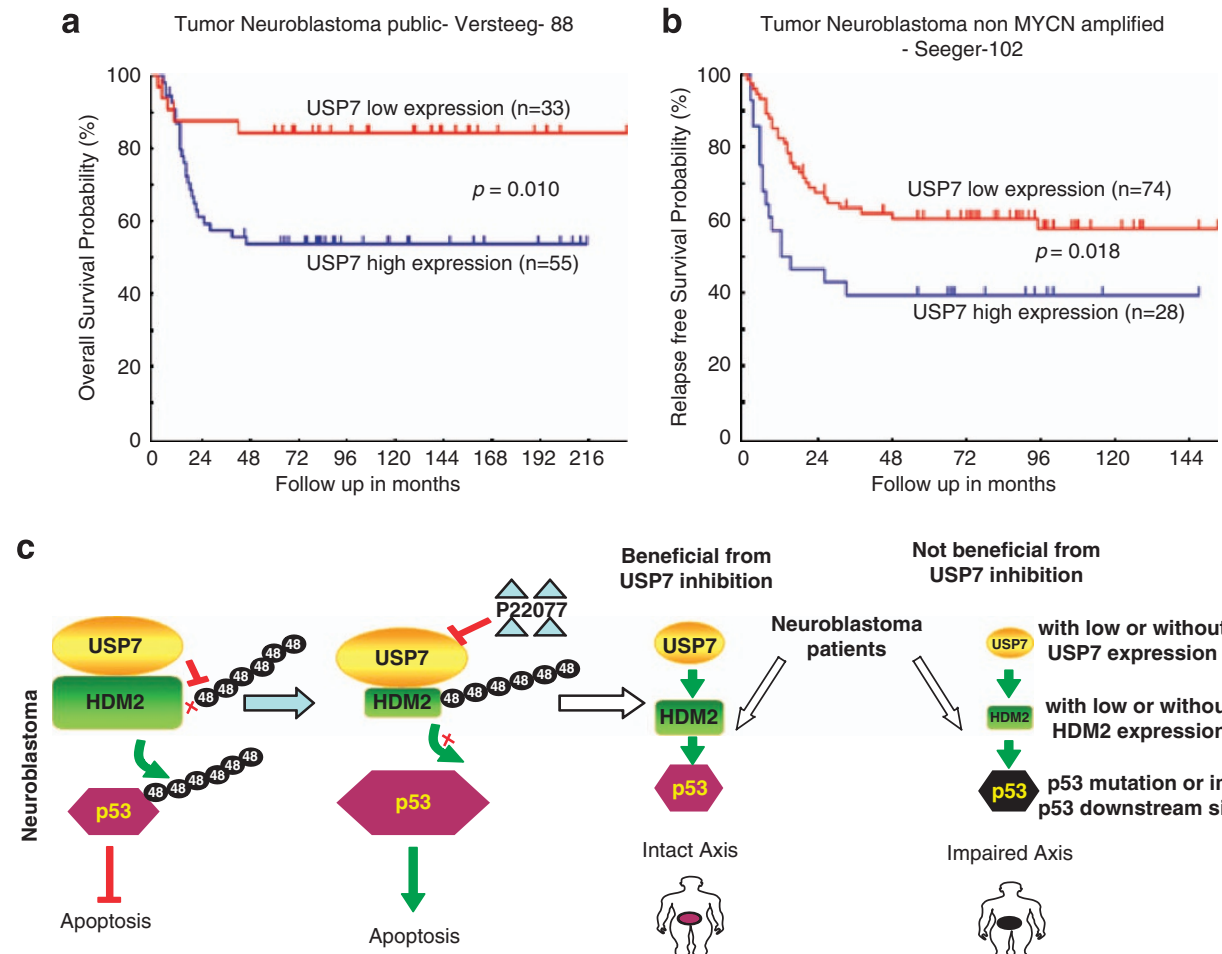

\section{Beneficial from Not beneficial from USP7 inhibition USP7 inhibition}

Figure 7 Outcomes of NB patients based on USP7 gene expression. The R2 gene expression databases (R2: http://r2.amc.nl) were evaluated for outcomes of NB patients, and Kaplan-Meier survival curves were generated. (a) Estimated overall survival for patients who have tumors with high USP7 gene expression (blue; $n=55$ ) and low USP7 gene expression (red; $n=33$ ). (b) Estimated relapse-free survival for patients who have tumors with high USP7 gene expression (blue; $n=28$ ) and low USP7 gene expression (red; $n=74$ ). (c) A working model to show that USP7 de-ubiquitinates and stabilizes HDM2. HDM2 further ubiquitinates p53 and promotes p53 degradation. P22077-mediated inhibition of USP7 function results in HDM2 degradation and p53 reactivation in NB. NB patients with an intact USP7-HDM2-p53 axis will be beneficial from P22077 treatment as either a single antitumor drug or an effective adjunct to current chemotherapeutic regimens

1640 containing $20 \%$ heat-inactivated FBS, $100 \mathrm{units} / \mathrm{ml}$ penicillin, $100 \mu \mathrm{g} / \mathrm{ml}$ streptomycin, and $2 \mathrm{mM}$ glutamine. All cells were grown in a humidified incubator containing $5 \% \mathrm{CO}_{2}$ at $37^{\circ} \mathrm{C}$. NB- 19 and LA-N-6 were kindly provided by Dr. A Davidoff (St. Judes's Children's Research Hospital, Memphis, TN, USA) and Dr. R Seeger (Children's Hospital of Los Angeles, Los Angeles, CA, USA), respectively. The SK-N-AS cell line with stable expression of luciferase was generated by transfection with a pcDNA3 luciferase expression plasmid into cells. A stable cell line was established after 10 days of $800 \mu \mathrm{g} / \mathrm{ml} \mathrm{G418} \mathrm{(Enzo} \mathrm{Life}$ Sciences, Farmingdale, NY, USA) selection.

Cell viability assay. Cell viability assays were assessed using the Cell Counting Kit-8 (CCK-8, WST-8[2-(2-methoxy-4-nitrophenyl)-3-(4-nitrophenyl)-5(2,4-disulfophenyl)-2 $\mathrm{H}$-tetrazolium, monosodium salt]) (Dojindo Laboratories, Rockville, MA, USA) following the manufacturer's instructions. Cells were seeded in 96-well flat-bottomed plates at the density of $1 \times 10^{4}$ per well. After $24 \mathrm{~h}$ of incubation at $37^{\circ} \mathrm{C}$, increasing concentrations of P22077, Dox, VP-16, or their combinations were added to the wells. Twenty-four hours later, $10 \mu$ l of CCK-8 was added into each well and after $1 \mathrm{~h}$ of incubation, the absorbance was measure at $450 \mathrm{~nm}$ using the microplate reader. Each experiment was performed in replicates of six. Background reading of media only was used to normalize the results.

Immunoblotting assay. Cells were washed with ice cold PBS and lysed using cold RIPA buffer ( $25 \mathrm{mM}$ HEPES at pH 7.7, $135 \mathrm{mM} \mathrm{NaCl}, 1 \%$ Triton X-100, $25 \mathrm{mM} \beta$-glycerophosphate, $0.1 \mathrm{mM}$ sodium orthovanadate, $0.5 \mathrm{mM}$ phenylmethylsulfonylfluoride, $1 \mathrm{mM}$ dithiothreitol, $10 \mu \mathrm{g} / \mathrm{ml}$ aprotinin, $10 \mu \mathrm{g} / \mathrm{ml}$ leupeptin, $1 \mathrm{mM}$ benzamidine, $20 \mathrm{mM}$ disodium p-nitrophenylphosphate, and phosphatase inhibitor cocktail 2 and 3) (P5726 and P0044) (Sigma). After measuring the protein concentrations using Bradford reagent (Bio-Rad, Hercules, CA, USA), the wholecell lysates were subjected to sodium dodecyl sulfate (SDS) polyacrylamide gel electrophoresis (PAGE), transferred to polyvinylidence fluoride (PVDF) membrane
(Bio-Rad), and then probed with the indicated primary antibodies overnight at $4{ }^{\circ} \mathrm{C}$. After incubation with the respective horse radish peroxidase (HRP)-conjugated secondary antibodies against mouse or rabbit for $1 \mathrm{~h}$ at $\mathrm{RT}\left(25^{\circ} \mathrm{C}\right)$, the membranes were visualized using the ECL-Plus Western detection system (GE Health Care, Buckinghamshire, UK).

Propidium iodide staining assay. Cells were exposed to different concentrations of P22077, Dox, VP-16, or DMSO for $24 \mathrm{~h}$. Cells were trypsinized, resuspended in RPMI 1640 medium, centrifuged at $400 \times g$ for 5 min at $4^{\circ} \mathrm{C}$. Cells were resuspended and washed with cold PBS twice. Finally, non-fixed cells were resuspended in $1 \times$ binding buffer (51-66121E) (BD Biosciences, San Jose, CA, USA) at a concentration of $1 \times 10^{6}$ cells per $\mathrm{ml}$. Five microliters of propidium iodide (PI) staining solution (51-66211E) (BD Biosciences) was added to each tube containing $100 \mu \mathrm{l}$ of non-fixed suspended cells and incubated with cells for $15 \mathrm{~min}$ at RT. The samples were then analyzed by flow cytometry within $1 \mathrm{~h}$ after the addition of $400 \mu \mathrm{l}$ of $1 \times$ binding buffer. PI-positive cells were considered as apoptotic cells, which are permeable to PI owing to the loss of membrane integrity. Unstained cells were used as the negative control and untreated cells were used as a control to treated cells.

Cytotoxic effect of P22077 on NB cell proliferation. Cells with or without luciferase expression were seeded in 48-well or 6-well plates at appropriate concentrations. After incubation for $24 \mathrm{~h}$, cells were treated with 0,10 , or $20 \mu \mathrm{M}$ of P22077 for $24 \mathrm{~h}$ at $37^{\circ} \mathrm{C}$. Cells were observed and photographed either by adding D-luciferin (Caliper Life Sciences, Hopkinton, MA, USA) into cells followed by bioluminescence imaging or by the optical microscope.

Effect of P22077 on NB growth in an orthotopic mouse model. The orthotopic NB mouse model was established as previously described. ${ }^{43}$ Briefly, $1.5 \times 10^{6}$ human IMR-32, SH-SY5Y, or NGP cells with luciferase expression were surgically injected into the left renal capsule of 
5-week-old female NCR nude mice. IMR-32, SH-SY5Y, and NGP-derived xenografts were allowed to grow for $\sim 2-3$ weeks before randomizing the mice into a control group and a P22077 treatment group. Each group consisted of three or six mice. Animals were treated with DMSO or P22077 by intraperitoneal (i.p.) injection every day for 12, 14, or 21 days. At the end of the experiments, all mice were killed. Tumors and the right side control kidneys were resected, weighed, and photographed. All mice were housed in a pathogen-free environment and handled in strict accordance to institutional protocol.

Statistical analysis. Two-tailed Student's $t$-test was used to determine the statistical significance of the in vitro drug treatment experiments. Two- or onetailed Student's $t$-test was used to determine the statistical significance of tumor sizes between the control and treatment groups. All values are presented as the mean \pm standard deviation (S.D.). A $P$-value of less than 0.05 was considered statistically significant.

\section{Conflict of Interest}

The authors declare no conflict of interest.

Acknowledgements. We are very grateful to Dr. A Davidoff and Dr. R Seeger for providing the NB cell lines described in this paper. We also thank Kristine Yang for editing our manuscript. This work was supported by the NIH-NINDS grant 1R01NS072420 (to JY). Jin Cheng is a recipient of China Scholarship Council fellowship grant.

1. Hoehner JC, Gestblom C, Hedborg F, Sandstedt B, Olsen L, Pahlman S. A developmental model of neuroblastoma: differentiating stroma-poor tumors' progress along an extra-adrenal chromaffin lineage. Lab Invest 1996; 75: 659-675.

2. Maris JM. Recent advances in neuroblastoma. N Engl J Med 2010; 362: 2202-2211.

3. Laverdiere C, Cheung NK, Kushner BH, Kramer K, Modak S, LaQuaglia MP et al. Long-term complications in survivors of advanced stage neuroblastoma. Pediatr Blood Cancer 2005; 45: 324-332.

4. Maris JM, Hogarty MD, Bagatell R, Cohn SL. Neuroblastoma. Lancet 2007; 369: 2106-2120.

5. Seeger RC. Immunology and immunotherapy of neuroblastoma. Semin Cancer Biol 2011; 21: 229-237.

6. Chesler L, Weiss WA. Genetically engineered murine models-contribution to our understanding of the genetics, molecular pathology and therapeutic targeting of neuroblastoma. Semin Cancer Biol 2011; 21: 245-255.

7. Pugh TJ, Morozova O, Attiyeh EF, Asgharzadeh S, Wei JS, Auclair D et al. The genetic landscape of high-risk neuroblastoma. Nat Genet 2013; 45: 279-284.

8. Pant V, Quintas-Cardama A, Lozano G. The p53 pathway in hematopoiesis: lessons from mouse models, implications for humans. Blood 2012; 120: 5118-5127.

9. Zilfou JT, Lowe SW. Tumor suppressive functions of p53. Cold Spring Harb Perspect Biol 2009; 1: a001883.

10. Meek DW. Tumour suppression by p53: a role for the DNA damage response? Nat Rev Cancer 2009; 9: 714-723.

11. Dai C, Gu W. p53 post-translational modification: deregulated in tumorigenesis. Trends Mol Med 2010; 16: 528-536.

12. Hainaut $\mathrm{P}$, Hollstein M. p53 and human cancer: the first ten thousand mutations. Adv Cancer Res 2000; 77: 81-137.

13. Becker K, Marchenko ND, Maurice M, Moll UM. Hyperubiquitylation of wild-type p53 contributes to cytoplasmic sequestration in neuroblastoma. Cell Death Differ 2007; 14: 1350-1360.

14. Hosoi G, Hara J, Okamura T, Osugi Y, Ishihara S, Fukuzawa M et al. Low frequency of the p53 gene mutations in neuroblastoma. Cancer 1994; 73: 3087-3093.

15. Fesik SW. Promoting apoptosis as a strategy for cancer drug discovery. Nat Rev Cancer 2005; 5: 876-885.

16. Hogarty MD. The requirement for evasion of programmed cell death in neuroblastomas with MYCN amplification. Cancer Lett 2003; 197: 173-179.

17. Goldman SC, Chen CY, Lansing TJ, Gilmer TM, Kastan MB. The p53 signal transduction pathway is intact in human neuroblastoma despite cytoplasmic localization. Am J Pathol 1996; 148: 1381-1385.
18. Wade M, Li YC, Wahl GM. MDM2, MDMX and p53 in oncogenesis and cancer therapy. Nat Rev Cancer 2013; 13: 83-96.

19. Kruse JP, Gu W. Modes of p53 regulation. Cell 2009; 137: 609-622.

20. Buschmann T, Fuchs SY, Lee CG, Pan ZQ, Ronai Z. SUMO-1 modification of Mdm2 prevents its self-ubiquitination and increases Mdm2 ability to ubiquitinate p53. Cell 2000; 101: 753-762.

21. Fuchs SY, Adler V, Buschmann T, Wu X, Ronai Z. Mdm2 association with $\mathrm{p} 53$ targets its ubiquitination. Oncogene 1998; 17: 2543-2547.

22. de Rozieres S, Maya R, Oren M, Lozano G. The loss of mdm2 induces p53-mediated apoptosis. Oncogene 2000; 19: 1691-1697.

23. Jones SN, Roe AE, Donehower LA, Bradley A. Rescue of embryonic lethality in Mdm2-deficient mice by absence of p53. Nature 1995; 378: 206-208.

24. Montes de Oca Luna R, Wagner DS, Lozano G. Rescue of early embryonic lethality in mdm2-deficient mice by deletion of p53. Nature 1995; 378: 203-206.

25. Li M, Brooks CL, Kon N, Gu W. A dynamic role of HAUSP in the p53-Mdm2 pathway. Mol Cell 2004; 13: 879-886.

26. Kon N, Kobayashi Y, Li M, Brooks CL, Ludwig T, Gu W. Inactivation of HAUSP in vivo modulates p53 function. Oncogene 2010; 29: 1270-1279.

27. Altun M, Kramer HB, Willems LI, McDermott JL, Leach CA, Goldenberg SJ et al. Activity-based chemical proteomics accelerates inhibitor development for deubiquitylating enzymes. Chem Biol 2011; 18: 1401-1412.

28. Nakamura $Y$, Ozaki T, Niizuma H, Ohira M, Kamijo T, Nakagawara A. Functional characterization of a new p53 mutant generated by homozygous deletion in a neuroblastoma cell line. Biochem Biophys Res Commun 2007; 354: 892-898.

29. Fan $Y$, Cheng J, Vasudevan SA, Patel RH, Liang L, Xu X et al. TAK1 inhibitor 5Z-7-oxozeaenol sensitizes neuroblastoma to chemotherapy. Apoptosis 2013; 18: 1224-1234.

30. Mandinova A, Lee SW. The p53 pathway as a target in cancer therapeutics: obstacles and promise. Sci Transl Med 2011; 3: 64rv61.

31. Brown CJ, Cheok CF, Verma CS, Lane DP. Reactivation of p53: from peptides to small molecules. Trends Pharmacol Sci 2011; 32: 53-62.

32. Pishas KI, Al-Ejeh F, Zinonos I, Kumar R, Evdokiou A, Brown MP et al. Nutlin-3a is a potential therapeutic for ewing sarcoma. Clin Cancer Res 2011; 17: 494-504.

33. Yang Y, Ludwig RL, Jensen JP, Pierre SA, Medaglia MV, Davydov IV et al. Small molecule inhibitors of HDM2 ubiquitin ligase activity stabilize and activate p53 in cells. Cancer Cell 2005; 7: 547-559.

34. Barbieri E, Mehta P, Chen Z, Zhang L, Slack A, Berg S et al. MDM2 inhibition sensitizes neuroblastoma to chemotherapy-induced apoptotic cell death. Mol Cancer Ther 2006; 5: 2358-2365.

35. Patterson DM, Gao D, Trahan DN, Johnson BA, Ludwig A, Barbieri E et al. Effect of MDM2 and vascular endothelial growth factor inhibition on tumor angiogenesis and metastasis in neuroblastoma. Angiogenesis 2011; 14: 255-266.

36. Manfredi JJ. The Mdm2-p53 relationship evolves: Mdm2 swings both ways as an oncogene and a tumor suppressor. Genes Dev 2010; 24: 1580-1589.

37. Nicholson B, Marblestone JG, Butt TR, Mattern MR. Deubiquitinating enzymes as novel anticancer targets. Future Oncol 2007; 3: 191-199.

38. Daviet L, Colland F. Targeting ubiquitin specific proteases for drug discovery. Biochimie 2008; 90: 270-283.

39. Chauhan D, Tian Z, Nicholson B, Kumar KG, Zhou B, Carrasco R et al. A small molecule inhibitor of ubiquitin-specific protease-7 induces apoptosis in multiple myeloma cells and overcomes bortezomib resistance. Cancer Cell 2012; 22: 345-358.

40. Reverdy C, Conrath S, Lopez R, Planquette C, Atmanene C, Collura V et al. Discovery of specific inhibitors of human USP7/HAUSP deubiquitinating enzyme. Chem Biol 2012; 19: 467-477.

41. Zhang Y, Zhou L, Rouge L, Phillips AH, Lam C, Liu P et al. Conformational stabilization of ubiquitin yields potent and selective inhibitors of USP7. Nat Chem Biol 2013; 9: 51-58.

42. Colland F, Formstecher E, Jacq X, Reverdy C, Planquette C, Conrath S et al. Small-molecule inhibitor of USP7/HAUSP ubiquitin protease stabilizes and activates p53 in cells. Mol Cancer Ther 2009; 8: 2286-2295.

43. Patterson DM, Shohet JM, Kim ES. Preclinical models of pediatric solid tumors (neuroblastoma) and their use in drug discovery. Curr Protoc Pharmacol 2011; Chapter 14: Unit 1417

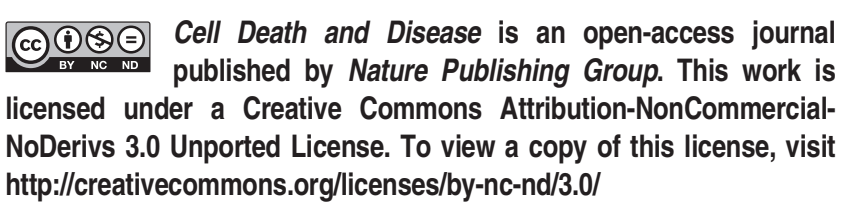

